\title{
In the flow: a finely controlled approach to catalyzed nanowire growth
}

\author{
William E Buhro \\ NPG Asia Materials (2014) 6, e83; doi:10.1038/am.2013.77; Published online 7 February 2014
}

A breakthrough in semiconductor nanowire synthesis that allows fine control over axial heterostructuring was recently advanced by Hollingsworth and co-workers. ${ }^{1}$ The report also reveals fascinating mechanistic aspects of catalyzed nanowire growth.

Catalyzed wire or whisker growth was discovered by Wagner and Ellis in 1964. ${ }^{2}$ They found that gold droplets on a silicon substrate catalyze silicon wire growth under chemical-vapor-deposition conditions. Gaseous precursors react at the golddroplet surfaces, depositing silicon into solution within the gold droplets. The droplets become supersaturated, inducing precipitation of crystalline silicon upon the substrate. As precipitation occurs only at the droplet-silicon interfaces, the silicon crystallites acquire pseudo-cylindrical wire morphologies as they grow upward from the substrate. The gold-catalyst droplets rise elevator-like from the substrate, riding upon the tips of the growing wires. Wagner and Ellis ${ }^{2}$ named this method 'vaporliquid-solid' or 'VLS' growth after the three participating phases: the vaporous precursors, liquid catalyst droplets and solid silicon wires.

The VLS synthesis was subsequently adapted to a wide range of wire compositions, and to nanometerscale wire diameters. ${ }^{3}$ A solution-based analog of VLS growth was also developed, in which precursors are delivered to the catalyst droplets in an organicsolvent phase, and nanowires grow in a solvent dispersion. By analogy, this method was named 'solution-liquid-solid' or 'SLS' growth.,

Certain advantages accrue to the SLS method, principally that it is conducted in a cocktail of surface ligands, which control growth, passivate electronic surface traps and provide nanowire dispersibility. However, a major drawback to SLS growth is that it is typically a batch process that does not readily support heterostructure formation. Many nanowire applications require heterojunctions, and therefore nanowire heterostructuring in which the composition is varied in segments along the axial dimension. In contrast, VLS growth supports axial heterostructuring by its easy variation of the gaseous precursors delivered.

Department of Chemistry, Washington University, St Louis, MO, USA

E-mail: buhro@wustl.edu

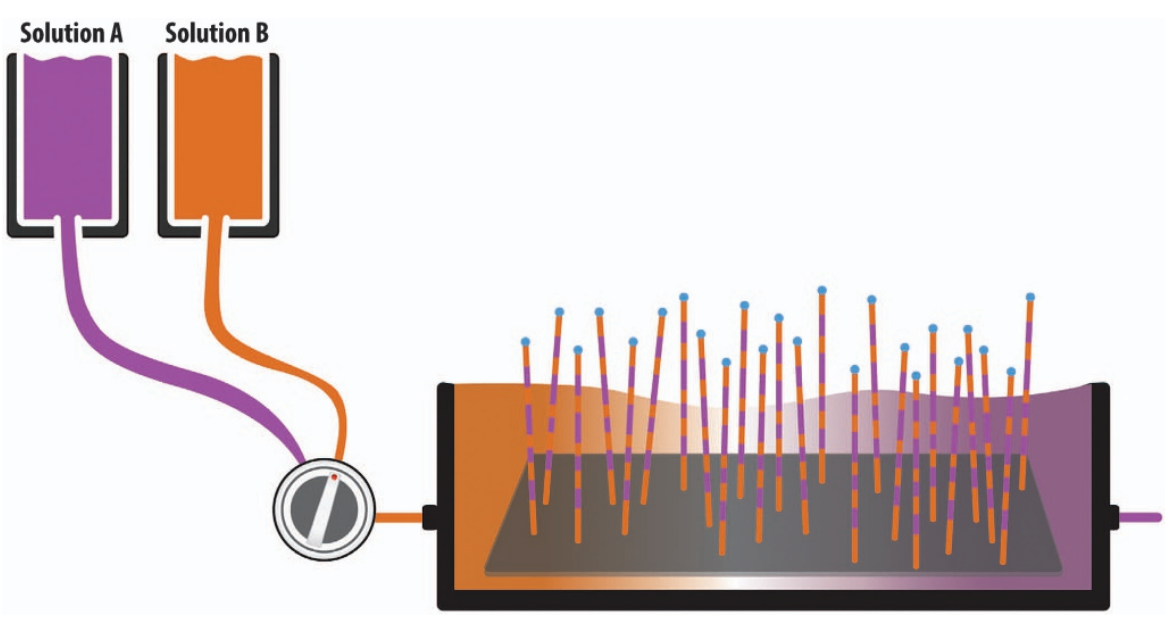

Figure 1 Axially heterostructured nanowires are grown in a microfluidic reactor by the flow-SLS method. ${ }^{1}$ Precursors corresponding to compositions $A$ and $B$ are alternately passed through the reactor, such that excess precursors and byproducts are continuously removed. Thus, alternating axial segments of semiconductors $A$ and $B$ are produced. Note the catalyst droplets attached to the growing tips of the nanowires (courtesy of Jennifer Hollingsworth).

Hollingsworth and co-workers ${ }^{1}$ have combined the advantages of the SLS and VLS approaches in a new flow-SLS method. Here heterostructured (CdSe$\mathrm{ZnSe}$ ) nanowires are grown in a microfluidic reactor, in which the precursor flow can be abruptly varied in an alternating manner between solutions $\mathrm{A}$ and $\mathrm{B}$ (Figure 1), resulting in nanowires having to date as many as eight alternating axial segments.

Moreover, the flow-SLS method slows the growth kinetics sufficiently to provide important new mechanistic insights. The results establish that nanowire growth occurs both by direct precursor impingement on the catalyst droplet, and by impingement on the nanowire sidewall with precursor diffusion along the wire to the catalyst droplet at the tip. In the latter case, nanowires elongate more rapidly as they lengthen and thus their sidewall area increases.

Further work on the flow-SLS method is required to achieve finer diameter control, to establish the sharpness of the heterojunctions formed, and to determine the electrical and optical properties of the heterostructured nanowires so produced.
1 Laocharoensuk, R., Palaniappan, K., Smith, N. A., Dickerson, R. M., Werder, D. J., Baldwin, J. K. \& Hollingsworth, J. A. Flow-based solution-liquid-solid nanowire synthesis. Nat. Nanotechnol. 8, 660-666 (2013).

2 Wagner, R. S. \& Ellis, W. C. Vapor-liquid-solid mechanism of single crystal growth. Appl. Phys. Lett. 4, 89-90 (1964).

3 Yang, P., Yan, R. \& Fardy, M. Semiconductor nanowire: What's Next? Nano. Lett. 10, 1529-1536 (2010).

4 Trentler, T. J., Hickman, K. M., Goel, S. C., Viano, A. M., Gibbons, P. C. \& Buhro, W. E. Solution-liquid-solid growth of crystalline III-V semiconductors: an analogy to vapor-liquid-solid growth. Science 270, 1791-1794 (1995).

5 Wang, F., Dong, A., Sun, J., Tang, R., Yu, H. \& Buhro, W. E. Solution-liquid-solid growth of semiconductor nanowires. Inorg. Chem. 45, 7511-7521 (2006).

cc) (i) $($ ) $९$ This work is licensed under a creative Commons AttributionNonCommercial-NoDerivs 3.0 Unported License. To view a copy of this license, visit http://creativecommons.org/licenses/bync-nd/3.0/ 